\section{SOI: $1.1 / \mathrm{TAS} \quad$ DOI: $10.15863 / \mathrm{TAS}$ International Scientific Journal Theoretical \& Applied Science}

p-ISSN: 2308-4944 (print) $\quad$ e-ISSN: 2409-0085 (online)

Year: $2016 \quad$ Issue: 2 Volume: 34

Published: 29.02.2016 $\underline{\text { http://T-Science.org }}$
Denis Alexandrovich Chemezov Master of Engineering and Technology, Corresponding member of International Academy of Theoretical and Applied Sciences, Lecturer of Vladimir Industrial College, Russian Federation chemezov-da@yandex.ru

SECTION 7. Mechanics and machine construction.

\title{
THE PARAMETERS OF THE GAS TURBINE BLADE WHEN CHANGING OF THE RATIO OF THE OUTPUT/INPUT RADIUS OF THE HUB
}

Abstract: The article is presented the calculation of parameters of the blade and defined the character of working changes of the gas turbine when the ratio of the output/input radius of the hub 0.2, 0.3 and 0.4.

Key words: a blade, a gas turbine, velocity, Mach number, pressure, an angle, a hub.

Language: Russian

Citation: Chemezov DA (2016) THE PARAMETERS OF THE GAS TURBINE BLADE WHEN CHANGING OF THE RATIO OF THE OUTPUT/INPUT RADIUS OF THE HUB. ISJ Theoretical \& Applied Science, 02 (34): 75-85.

Soi: http://s-o-i.org/1.1/TAS-02-34-13 Doi: crossef http://dx.doi.org/10.15863/TAS.2016.02.34.13

\section{ПАРАМЕТРЫ ЛОПАСТИ ГАЗОВОЙ ТУРБИНЫ ПРИ ИЗМЕНЕНИИ ОТНОШЕНИЯ ВЫХОДНОГО/ВХОДНОГО РАДИУСОВ СТУПИЦЫ}

Аннотация: В статье представлен расчет параметров лопасти и определен характер изменения работы газовой турбины при отношении выходного/входного радиусов ступицы 0.2, 0.3 и 0.4.

Ключевые слова: лопасть, газовая турбина, скорость, число Маха, давление, угол, ступица.

Газовая турбина применяется для преобразования энергии сжатого и нагретого газа в механическую работу (вращение вала турбины) [1]. Крутящий момент на главном валу газовой турбины создается за счет вращающихся деталей и в частности рабочих лопастей (лопаток), на которые действует газовый поток.

Аэродинамический профиль лопасти газовой турбины имеет сложную конфигурацию. Лопасть газовой турбины состоит из следующих частей: хвоста для крепления детали в ободе диска, рабочей части, находящейся под действием движущегося потока газа, и вершины для закрепления ленточного бандажа (связывание нескольких лопастей) [2]. Рабочая часть лопасти состоит из ряда элементов: наружной и внутренней поверхностей, входной и выходной кромок, утолщения, отверстия под скрепление, наружной и внутренней галтелей. Также рабочая часть лопасти характеризуется наружным и внутренним профилями сечения [3].

Геометрическая форма и величины размеров рабочей лопасти влияют на производительность газовой турбины. Анализ характера работы газовой турбины при изменении геометрических параметров лопасти можно выполнить на этапе проектирования посредством специальных компьютерных программ.

Проектировались 3 радиальные газовые турбины с различными профилями рабочих лопастей в модуле Vista RTD (предварительный расчет радиальных турбин) программного комплекса Vista TF (экспресс-анализ характеристик течения газа в турбомашине). В качестве переменного параметра принято отношение выходного/входного радиусов ступицы (R3hub/R2) газовой турбины величинами $0.2,0.3$ и 0.4 . Проектирование лопасти газовой турбины осуществлялось для одной ступени. Исходными данными являлись условия эксплуатации газовой турбины и геометрические размеры ее основных элементов.

Условия эксплуатации (аэродинамические характеристики) радиальной газовой турбины при различных R3hub/R2 представлены в табл. 1.

Геометрические размеры сопла и рабочего колеса радиальной газовой турбины при различных R3hub/R2 представлены в табл. 2. 


\begin{tabular}{|c|c|c|c|c|c|c|}
\hline Impact Factor: & $\begin{array}{l}\text { ISRA (India) } \\
\text { ISI (Dubai, UAF } \\
\text { GIF (Australia) } \\
\text { JIF }\end{array}$ & $\begin{array}{l}=1.344 \\
=0.829 \\
=0.564 \\
=1.500\end{array}$ & $\begin{array}{l}\text { SIS (USA) } \\
\text { PИНЦ (Russia) } \\
\text { ESJI (KZ) } \\
\text { SJIF (Morocco) }\end{array}$ & $\begin{array}{l}=0.912 \\
=0.179 \\
=1.042 \\
=2.031\end{array}$ & $\begin{array}{l}\text { ICV (Poland) } \\
\text { PIF (India) }\end{array}$ & $\begin{array}{l}=6.630 \\
=1.940\end{array}$ \\
\hline
\end{tabular}

Таблица 1

Аэродинамические характеристики.

\begin{tabular}{|c|c|c|c|}
\hline \multicolumn{4}{|l|}{ Условия эксплуатации } \\
\hline Отночение выходного/входного радиусов ступицы (R3hub/R2) & 0.2 & 0.3 & 0.4 \\
\hline Температура торможения на входе, К (Т01) & & 350 & \\
\hline Давление торможения на входе, кПа (Р01) & & 152 & \\
\hline Удельный массовый расход, кг/с (Mass) & & 0.2 & \\
\hline Степень расширения газа /полная-полная/ (Exp tt) & & 2.917 & \\
\hline Частота вращения, мин $^{-1}(\mathrm{~N})$ & & 42000 & \\
\hline Отношение скоростей (U/C t-t) & & 0.7 & \\
\hline \multicolumn{4}{|l|}{ КПД ступени } \\
\hline Установка пользователя & & 0.8 & \\
\hline \multicolumn{4}{|l|}{ Свойства газа (жидкости) } \\
\hline Удельная теплоемкость при постоянном давлении, Дж /(кг · К) (Cp) & & 1004 & \\
\hline Газовая постоянная, Дж /(кг · К) $(\mathrm{R})$ & & 287 & \\
\hline \multicolumn{4}{|l|}{ Угль отклонения потока } \\
\hline \multicolumn{4}{|l|}{ Входной угол } \\
\hline Относительный, град. & & -40.1 & \\
\hline \multicolumn{4}{|l|}{ Выходной угол } \\
\hline Абсолютный, град. & & 0 & \\
\hline
\end{tabular}

Геометрические размеры.

Таблица 2

\begin{tabular}{|c|c|c|c|}
\hline Отночение выходного/входного радиусов ступицы (R3hub/R2) & 0.2 & 0.3 & 0.4 \\
\hline \multicolumn{4}{|l|}{ Сопло } \\
\hline Средняя толщина лопасти в горловине, мм (Noz thk) & & 1 & \\
\hline Количество лопастей (Noz vanes) & & 10 & \\
\hline Проходное сечение горловины, мм² (Tht area) & & 780 & \\
\hline \multicolumn{4}{|l|}{ Рабочее колесо } \\
\hline Средняя толщина лопасти на выходе, мм (Imp thk) & & 1.5 & \\
\hline Количество лопастей (Imp vanes) & & 14 & \\
\hline Отношение выходного/входного радиусов обода (R3shr/R2) & & 0.65 & \\
\hline Длина по оси, \% & & 35 & \\
\hline
\end{tabular}

Энергия потока газа полностью преобразовывалась в давление на входе, торможение газа адиабатное и изоэнтропическое (тепловая характеристика набегающего потока) [4].
Результаты расчета производительности, скоростей течения газа и геометрических размеров элементов газовой турбины при различных R3hub/R2 представлены в табл. 3.

Таблица 3

\section{Результаты расчета.}

\begin{tabular}{|c|c|c|c|}
\hline Отночение выходного/входного радиусов ступицы (R3hub/R2) & 0.2 & 0.3 & 0.4 \\
\hline \multicolumn{4}{|l|}{ Производительность } \\
\hline Оценка полного статического изоэнтропического КПД ступени, \% (Eff) & \multicolumn{3}{|c|}{80} \\
\hline $\begin{array}{c}\text { Отношение выходной предельной относительной скорости к входной } \\
\text { относительной скорости (W3s/W2) }\end{array}$ & 2.367 & 2.395 & 2.467 \\
\hline $\begin{array}{c}\text { Отношение выходной предельной осевой скорости к входной } \\
\text { относительной скорости (Vax3/U2) }\end{array}$ & 0.181 & 0.21 & 0.269 \\
\hline Степень расширения /полная-статическая/ (Exp ts) & 2.973 & 2.992 & 3.041 \\
\hline $\begin{array}{c}\text { Функция течения/Функция течения с учетом величины дросселирования } \\
\text { (Q/Qchk) }\end{array}$ & 0.937 & 0.943 & 0.953 \\
\hline Mass $\cdot \sqrt{T_{05}} / P_{05}, \mathrm{\kappa} \Gamma \cdot \mathrm{K} / \mathrm{\kappa} П а(\operatorname{MrtT} / \mathrm{P}(\mathrm{e}-5))$ & \multicolumn{3}{|c|}{$2.462 \cdot 10^{-5}$} \\
\hline
\end{tabular}

ISPC Technology and Innovation,

Philadelphia, USA 


\begin{tabular}{|c|c|c|c|}
\hline Мощность, кВт (Pwr) & \multicolumn{3}{|c|}{14.822} \\
\hline Реакция (Rctn) & 0.583 & 0.59 & 0.607 \\
\hline $\begin{array}{c}\text { С - скорость струи на выходе; т. е. это скорость, которая может быть } \\
\text { получена если газ был изоэнтропически расширен (энтальпия } \\
\text { торможения на входе и выходе), определяется как: } C=\sqrt{2 \cdot \Delta H_{\text {isen }}}(\mathrm{U} / \mathrm{C} \text { ts) }\end{array}$ & 0.695 & 0.693 & 0.689 \\
\hline $\begin{array}{l}\text { Коэффициент нагрузки (dh/U2) } \\
\end{array}$ & \multicolumn{3}{|c|}{0.816} \\
\hline Коэффициент быстроходности (Ns) & 0.46 & 0.461 & 0.464 \\
\hline Температура, K (T3) & 274.7 & 274.2 & 272.9 \\
\hline Температура, К (Т03) & \multicolumn{3}{|c|}{276.2} \\
\hline Давление, кПа (Р3) & 51.13 & 50.81 & 49.98 \\
\hline Давление, кПа (Р03) & \multicolumn{3}{|c|}{52.11} \\
\hline КПД ступени /полный-статический/, \% (Stg ts) & 78.8 & 78.4 & 77.4 \\
\hline КПД ступени /полный-полный/, \% (Stg tt) & \multicolumn{3}{|c|}{80.0} \\
\hline КПД рабочего колеса /полный-статический/, \% (Imp ts) & 81.4 & 80.9 & 79.9 \\
\hline КПД рабочего колеса /полный-полный/, \% (Imp tt) & \multicolumn{3}{|c|}{82.6} \\
\hline \multicolumn{4}{|l|}{ Скорости на входе } \\
\hline Абсолютное число Маха (Mabs) & \multicolumn{3}{|c|}{0.712} \\
\hline Относительное число Maхa (Mrel) & \multicolumn{3}{|c|}{0.24} \\
\hline Окружная скорость лопасти, м/с (U2) & \multirow{2}{*}{\multicolumn{3}{|c|}{$\begin{array}{c}301.31 \\
254.596\end{array}$}} \\
\hline Абсолютная скорость, м/с (V2) & \multirow{2}{*}{\multicolumn{3}{|c|}{$\frac{254.596}{85.919}$}} \\
\hline Относительная скорость, м/с (W2) & & & \\
\hline Абсолютная скорость закрутки потока, м/с (Vw2) & \multicolumn{3}{|c|}{245.967} \\
\hline Радиальная скорость, м/с (Vr) & \multicolumn{3}{|c|}{65.721} \\
\hline Абсолютный угол потока /радиальная ось/, град. (Alpha2) & \multicolumn{3}{|c|}{75.04} \\
\hline Относительный угол потока /радиальная ось/, град. (Beta2) & \multicolumn{3}{|c|}{-40.1} \\
\hline \multicolumn{4}{|l|}{ Выходные скорости на ободе } \\
\hline Абсолютное число Маха (Mabs) & 0.165 & 0.19 & 0.245 \\
\hline Относительное число Маха (Mrel) & 0.612 & 0.62 & 0.64 \\
\hline Окружная скорость лопасти, м/с (U3) & \multicolumn{3}{|c|}{195.851} \\
\hline Абсолютная скорость, м/с (V3) & 54.678 & 63.187 & 80.976 \\
\hline Относительная скорость, м/с (W3) & 203.34 & 205.79 & 211.93 \\
\hline Абсолютная скорость закрутки потока, м/c (Vw3) & \multicolumn{3}{|c|}{0} \\
\hline Осевая скорость, м/с (Vax3) & 54.678 & 63.187 & 80.976 \\
\hline Абсолютный угол потока /продольная ось/, град. (Alpha3) & \multicolumn{3}{|c|}{0} \\
\hline Относительный угол потока /продольная ось/, град. (Beta3) & -74.40 & -72.12 & -67.53 \\
\hline \multicolumn{4}{|l|}{ Геометрические размеры рабочего колеса } \\
\hline Диаметр входа, мм (d2) & \multicolumn{3}{|c|}{$\begin{array}{c}137.014 \\
6191\end{array}$} \\
\hline Ширина концевой кромки, мм (Tip width) & \multicolumn{3}{|c|}{6.191} \\
\hline Входной диаметр ступицы, мм (d3hub) & 27.403 & 41.104 & 54.806 \\
\hline Входной диаметр обода, мм (d3shr) & \multicolumn{3}{|c|}{89.059} \\
\hline $\begin{array}{l}\text { Отношение входного диаметра к предельному выходному диаметру } \\
\text { (d2/d3rms) }\end{array}$ & 2.08 & 1.975 & 1.853 \\
\hline Предельный относительный угол потока, град. (Beta3rms) & -69.32 & -67.49 & -63.52 \\
\hline Относительный угол потока на ободе, град. (Beta3shr) & -74.40 & -72.12 & -67.53 \\
\hline Относительный угол потока на ступице, град. (Beta3hub) & -47.78 & -55.04 & -56.10 \\
\hline \multicolumn{4}{|l|}{ Геометрия сопла } \\
\hline $\begin{array}{c}\text { Функция течения/Функция течения с учетом величины дросселирования } \\
\text { (Q/Qchk) }\end{array}$ & & 0.921 & \\
\hline $\begin{array}{c}\text { Диаметр окружности, измеренный по задним кромкам лопасти, мм (Inner } \\
\text { dia) }\end{array}$ & & 157.566 & \\
\hline Отношение A/R для безлопаточного сопла, мм (Vless A/R) & & 10.393 & \\
\hline Проходное сечение горловины, мм² (Tht area) & & 688 & \\
\hline
\end{tabular}

Изоэнтропический КПД - коэффициент, характеризующий эффективность работы газовой турбины работающей в условиях отсутствия теплообмена с окружающей средой. По значению коэффициента быстроходности определяют тип турбины. Число Маха является основным 
параметром движения газа (дозвуковым или сверхзвуковым) [5].

Изменение профиля лопасти при R3hub/R2 равном 0.4 приводит к увеличению более чем на $5 \%$ (относительно R3hub/R2 равного 0.2) значений следующих коэффициентов производительности газовой турбины: W3s/W2, Vax3/U2, Exp ts, Q/Qchk, Rctn, Ns. При этом уменьшаются величины коэффициента C, температуры и давления на выходе, КПД ступени (Stg ts) и рабочего колеса (Imp ts). Во всех случаях остаются неизменными параметры Eff, MrtT/P(e-5), Pwr, dh/U2, T03, P03, Stg tt и Imp tt.

Движение газа в турбине дозвуковое. Расчетное Mabs на выходе уменьшается от 3 до 4 раз по сравнению с Mabs на входе. Наибольшая величина Mabs определена на входе. Mrel увеличивается примерно в 2.5 раза на выходе для всеx R3hub/R2 радиальной газовой турбины. U изменяется в 1.5 раза с $301.3 \mathrm{M} / \mathrm{c}$ до $195.8 \mathrm{M} / \mathrm{c}$. V2 потока газа на входе составляет $254.6 \mathrm{~m} / \mathrm{c}$. V3 на выходе достигает значений 54.6 м/с при R3hub/R2 $=0.2$ и $80.9 \mathrm{~m} / \mathrm{c}$ при R3hub/R2 = 0.4 (увеличение на $50 \%)$. Vw газа прямо пропорциональна углу поворота потока [6]. Расчетная величина Vw2 составляет 246 м/с, а на выходе равна нулю. В соответствии с конструкцией турбины на входе течение газа происходит в радиальном направлении с неизменной скоростью, а на выходе - в осевом направлении. Значения осевых скоростей совпадают со значениями абсолютных скоростей при соответствующих R3hub/R2 газовой турбины.

Alpha газа изменяется на 75.04 град. на расстоянии от входа до выхода. Beta газа отрицательный, на выходе при R3hub/R2 = 0.2 имеет наибольшее значение (-74.40).

При одинаковом d2 и d3shr рабочего колеса радиальной газовой турбины, d3hub изменяется в диапазоне от 27.4 до 54.8 мм. Наименьший диаметр ступицы соответствует R3hub/R2 $=0.2$, наибольший $-\mathrm{R} 3 \mathrm{hub} / \mathrm{R} 2=0.4$. Разница $\mathrm{d} 2 / \mathrm{d} 3 \mathrm{rms}$ составляет $22.7 \%$ для R3hub/R2 = 0.2 и R3hub/R2 $=0.4$. По отношению площадь/радиус сопла $(\mathrm{A} / \mathrm{R})$ [7] производят выбор основных размеров газовой турбины. Расчетная величина Tht area сопла газовой турбины составило $688 \mathrm{~mm}^{2}$, что на 92

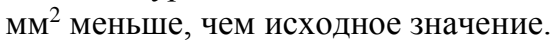

Расчетные профили лопастей газовой турбины (цифры 2 и 3 идентифицируют входную и выходную зоны) и треугольники скоростей при различных R3hub/R2 представлены на рис. 1.

Треугольник скоростей рабочего колеса газовой турбины представляет собой треугольник, построенный из векторов относительной и абсолютной скоростей газа и окружной скорости лопасти. В таком треугольнике вектор абсолютной скорости является геометрической суммой других векторов [8].

Контуры меридиональной скорости газа на лопасти газовой турбины при различных R3hub/R2 представлены на рис. 2.

Меридиональная скорость газа равна геометрической сумме радиальной и осевой составляющих скорости. Наибольшая меридиональная скорость газа наблюдается на середине лопасти газовой турбины при R3hub/R2 $=0.4$.

Контуры скорости вращения закрученного потока газа на лопасти газовой турбины при различных R3hub/R2 представлены на рис. 3.

Максимальная величина скорости вращения закрученного потока газа отмечена на входе. На выходе эта скорость уменьшается до $20-40$ м/с. Характер изменения скорости практически одинаков при различных R3hub/R2.

Контуры статического давления на лопасти газовой турбины при различных R3hub/R2 представлены на рис. 4.

Статическое давление не выражает условия равновесия газа. В соответствии с контурами снижение давления происходит в 2 раза (Р2 и Р3). Характер изменения статического давления практически одинаков при различных R3hub/R2.

Контуры статической температуры на лопасти газовой турбины при различных R3hub/R2 представлены на рис. 5.

Статическая температура представляет собой температуру газа в рассматриваемой точке. Разница температур на входе и на выходе - не менее 50 К. Характер изменения статической температуры практически одинаков при различных R3hub/R2.

Контуры коэффициента дросселирования [9] на лопасти газовой турбины при различных R3hub/R2 представлены на рис. 6.

Коэффициент дросселирования или коэффициент Джоуля-Томсона характеризует предел отношения изменения температуры газа к изменению его давления в изоэнтальпийном процессе. Наибольшие потери скорости воздушного потока наблюдаются на выходе лопасти газовой турбины при R3hub/R2 = 0.4.

Контуры погрешности решения на профиле лопасти газовой турбины при различных R3hub/R2 представлены на рис. 7.

Определяют величину погрешности расчета меридиональной скорости газа на лопасти газовой турбины в процентном выражении. Минимальная погрешность решения $( \pm 0.01 \%)$ найдена для R3hub/R2 = 0.3 и R3hub/R2 = 0.4.

Зависимости величины числа Маха на линии воздушного потока для различных зон лопасти газовой турбины от нормализованного М [10] представлены на рис. $8-10$. 
Нормализованное М рассчитывается из соотношения $\mathrm{M} / \mathrm{M}^{+}$, где $\mathrm{M}$ - адаптивное управление крутящим моментом турбины; $\mathrm{M}^{+}$оптимальное управление крутящим моментом турбины. Максимальное значение числа Маха отмечено на линии воздушного потока в зоне концевой кромки лопасти со стороны всасывания при $\mathrm{R} 3 \mathrm{hub} / \mathrm{R} 2=0.4$. Со стороны нагнетания на определенных интервалах нормализованного М наблюдаются отрицательные значения числа Maxa.

Зависимости величины нагрузочного параметра в межлопаточном канале [11] от нормализованного М представлены на рис. 11 .

Наибольшая нагрузка действует у ступицы при $\mathrm{R} 3 \mathrm{hub} / \mathrm{R} 2=0.2$. В средней части и $\mathrm{y}$ концевой кромки межлопаточного канала этот параметр уменьшается примерно в 4 раза.

Зависимости величины углов бета лопасти газовой турбины и отклонения потока газа от нормализованного М представлены на рис. 12.

С увеличением значения нормализованного М увеличивается и угол бета лопасти. При этом наибольшая величина угла на концевой кромке лопасти получена для R3hub/R2 $=0.2$, у ступицы - для R3hub/R2 = 0.4. Угол потока газа на концевой кромке и в средней части лопасти уменьшается при величинах нормализованного М $0-0.25$ и увеличивается на интервале $0.25-1$. У ступицы угол потока газа уменьшается на интервале $0-0.32$. Для $\mathrm{R} 3 \mathrm{hub} / \mathrm{R} 2=0.2$ на концевой кромке лопасти газовой турбины угол потока имеет минимальное значение -21 град., максимальное - 72 град.

Зависимости величины статического давления на лопасти газовой турбины в меридиональном сечении от нормализованного М представлены на рис. 13.

Характер снижения статического давления на лопасти газовой турбины представлен двумя фазами: первый - резкое уменьшение на интервале нормализованного М $0-0.35$ и второй - более медленное уменьшение на интервале 0.35 -1 .

Зависимости величины нагрузочных параметров [12] от нормализованного размаха лопасти газовой турбины представлены на рис. 14.
Число де Халлера равно отношению выходной/входной относительных скоростей. До $0.13(\mathrm{R} 3 \mathrm{hub} / \mathrm{R} 2=0.4)$ и $0.35(\mathrm{R} 3 \mathrm{hub} / \mathrm{R} 2=0.2)$ нормализованного размаха лопасти газовой турбины выходная относительная скорость меньше чем входная. Дальнейшее увеличение коэффициента нормализованного размаха лопасти газовой турбины приводит к увеличению выходной относительной скорости в 1.8 раза относительно входной. Лямбда - коэффициент работы. Определяется как отношение разности статических энтальпий пара в начале и в конце процесса расширения к окружной скорости лопасти газовой турбины. Минимальная величина коэффициента рассчитана для всех R3hub/R2 при нормализованном размахе лопасти газовой турбины равном 1 , максимальная - для $\mathrm{R} 3 \mathrm{hub} / \mathrm{R} 2=0.2$. Коэффициент восстановления идеального статического давления рассчитывается как отношение статических давлений в двух рассматриваемых сечениях потока газа. Увеличение коэффициента (1 при $\mathrm{R} 3 \mathrm{hub} / \mathrm{R} 2=0.2$ ) позволит снизить мощность, потребную для работы турбины. Величина параметра Цвайфеля позволяет определить рациональное количество лопастей, вес, стоимость и КПД газовой турбины. При величине числа Цвайфеля более 0.8 возрастают потери, связанные с уменьшением количества лопастей газовой турбины.

Зависимости величины углов падения и отклонения от нормализованного размаха лопасти газовой турбины представлены на рис. 15.

Угол падения протекающего газа зависит от поворота лопасти в одном из направлений. Для вcex R3hub/R2 этот угол изменяется на 3.2 град в диапазоне от -48.7 до -45.5 град. Угол отклонения - угол, под которым линия, касательная к нижнему краю лопасти, проходит относительно горизонтали. Расчетные значения угла могут быть положительными и отрицательными, в зависимости от величины нормализованного размаха лопасти газовой турбины. Наибольшие значения угла отклонения получены для $\mathrm{R} 3 \mathrm{hub} / \mathrm{R} 2=0.2$. 


\begin{tabular}{l|lrl|l|ll} 
& ISRA (India) & $=\mathbf{1 . 3 4 4}$ & SIS (USA) & $=\mathbf{0 . 9 1 2}$ & ICV (Poland) & $=\mathbf{6 . 6 3 0}$ \\
Impact Factor: & ISI (Dubai, UAE) $=\mathbf{0 . 8 2 9}$ & PUHU (Russia) $=\mathbf{0 . 1 7 9}$ & PIF (India) & $=\mathbf{1 . 9 4 0}$ \\
& GIF (Australia) & $\mathbf{0 . 5 6 4}$ & ESJI (KZ) & $=\mathbf{1 . 0 4 2}$ & & \\
& JIF & $=\mathbf{1 . 5 0 0}$ & SJIF (Morocco) $=\mathbf{2 . 0 3 1}$ & &
\end{tabular}

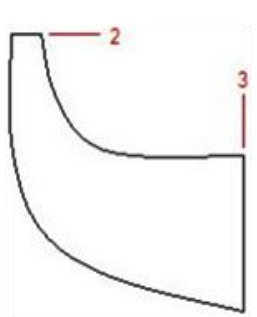

a)

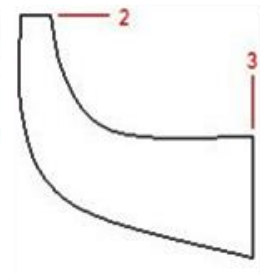

б)

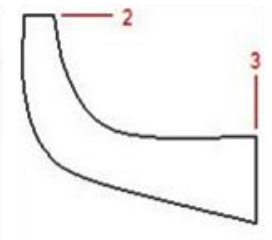

в)

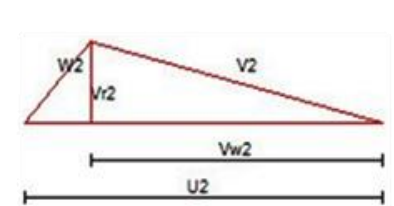

г)

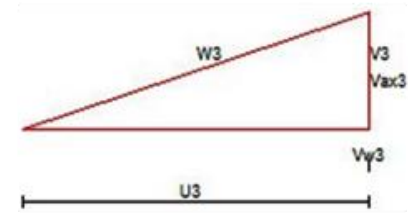

д)

Рисунок 1 - Профили лопасти газовой турбины (а - отношение выходного/входного радиусов ступицы 0.2; б - отношение выходного/входного радиусов ступицы 0.3 ; в - отношение выходного/входного радиусов ступицы 0.4) и треугольники скоростей (г - на входе; д - на выходе).

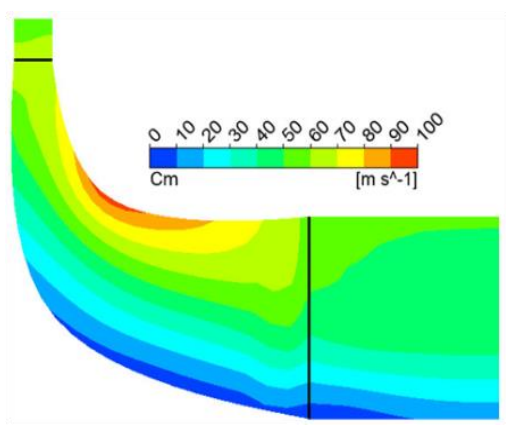

a)

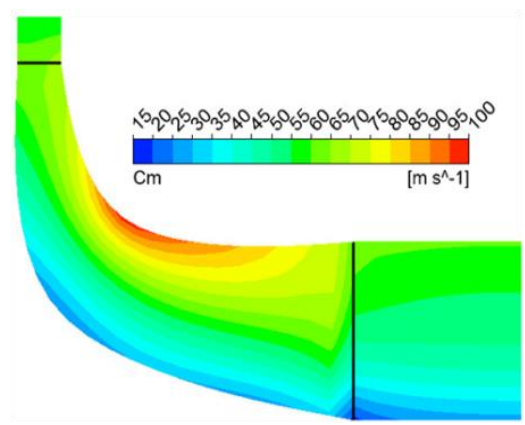

6)

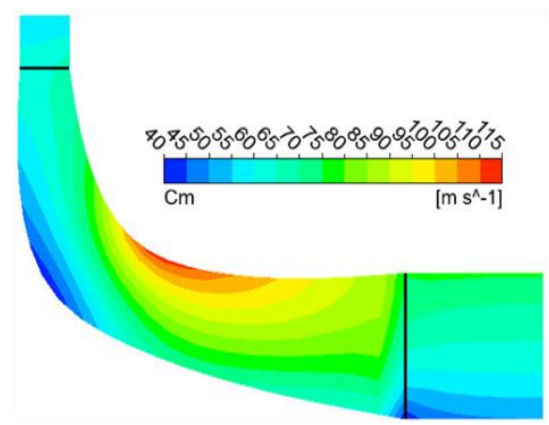

в)

Рисунок 2 - Контуры меридиональной скорости. Отношение выходного/входного радиусов ступицы

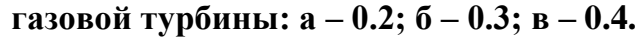

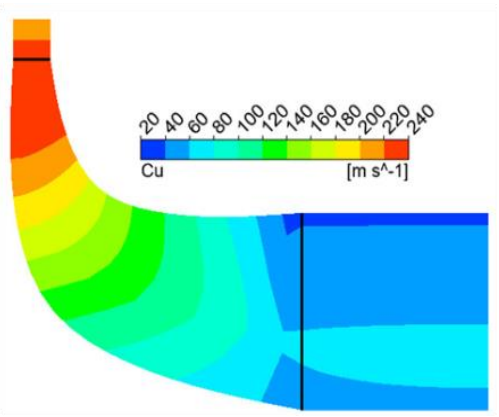

a)

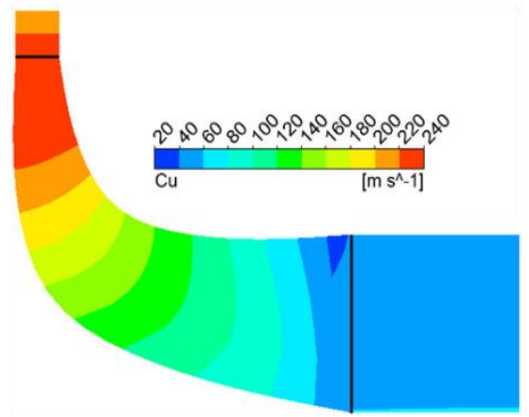

б)

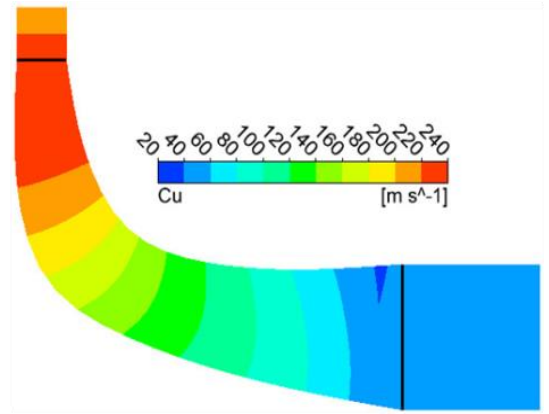

B)

Рисунок 3 - Контуры скорости вращения закрученного потока газа. Отношение выходного/входного

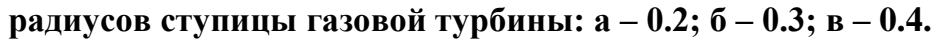

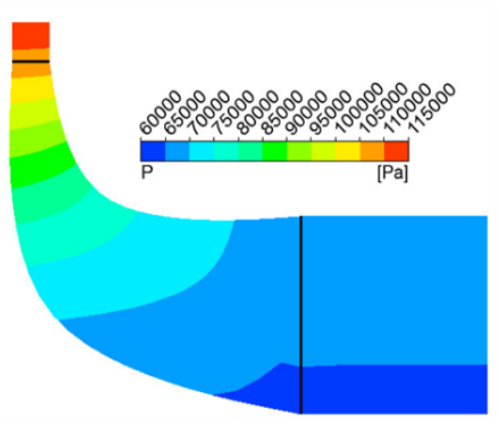

a)

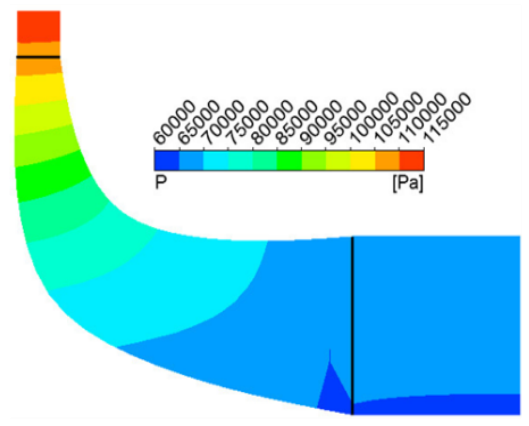

б)

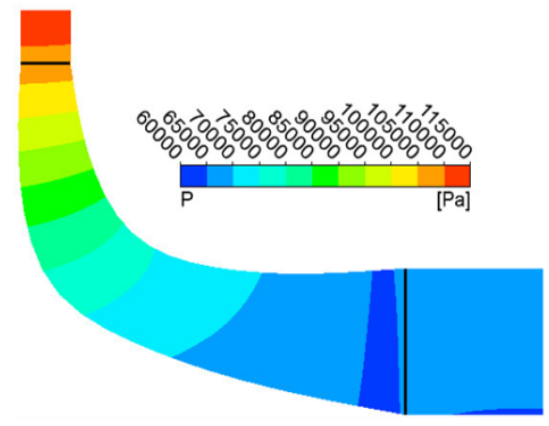

в)

Рисунок 4 - Контуры статического давления. Отношение выходного/входного радиусов ступицы

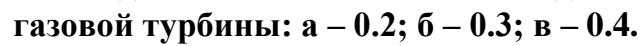




\begin{tabular}{l|lrl|l|ll} 
& ISRA (India) & $=\mathbf{1 . 3 4 4}$ & SIS (USA) & $=\mathbf{0 . 9 1 2}$ & ICV (Poland) & $=\mathbf{6 . 6 3 0}$ \\
Impact Factor: & ISI (Dubai, UAE) $=\mathbf{0 . 8 2 9}$ & PUHU (Russia) $=\mathbf{0 . 1 7 9}$ & PIF (India) & $=\mathbf{1 . 9 4 0}$ \\
& GIF (Australia) & $\mathbf{0 . 5 6 4}$ & ESJI (KZ) & $=\mathbf{1 . 0 4 2}$ & & \\
& JIF & $=\mathbf{1 . 5 0 0}$ & SJIF (Morocco) $=\mathbf{2 . 0 3 1}$ & &
\end{tabular}

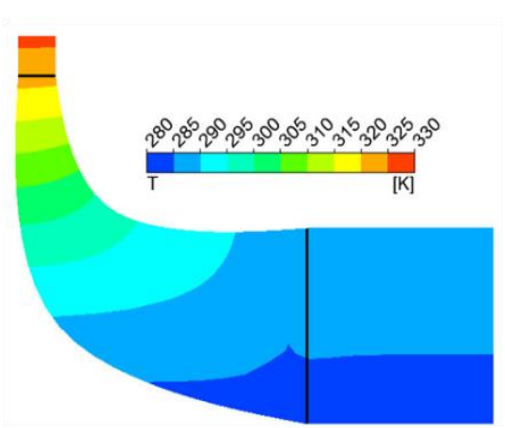

a)

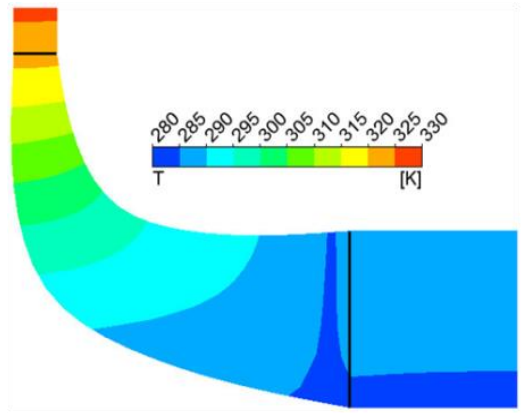

б)

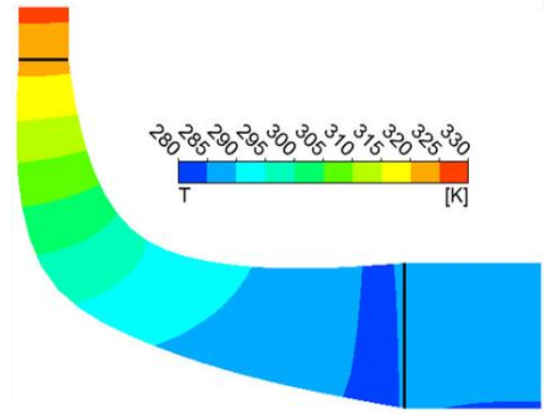

в)

Рисунок 5 - Контуры статической температуры. Отношение выходного/входного радиусов ступицы газовой турбины: $\mathbf{a}-0.2 ; \sigma-0.3 ; ~ в-0.4$.

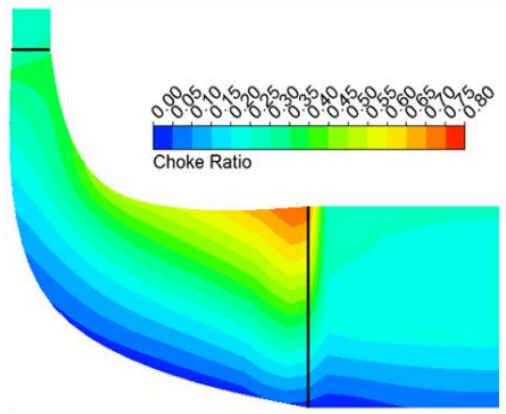

a)

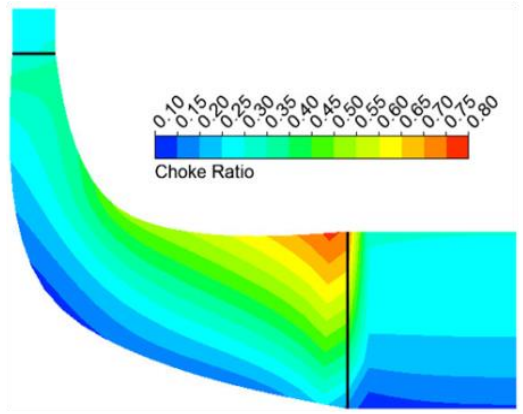

б)

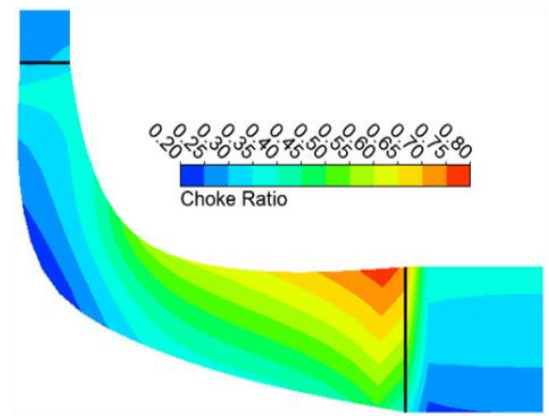

B)

Рисунок 6 - Контуры коэффициента дросселирования. Отношение выходного/входного радиусов ступицы газовой турбины: $а$ - 0.2; б-0.3; в-0.4.

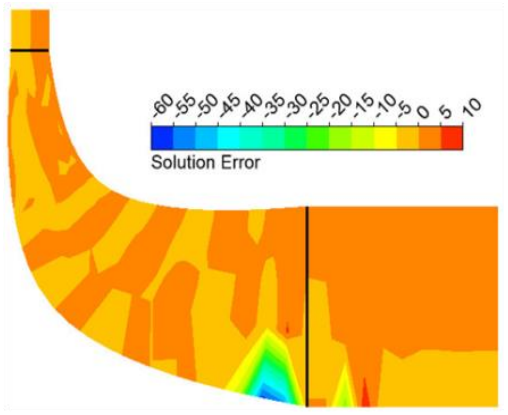

a)

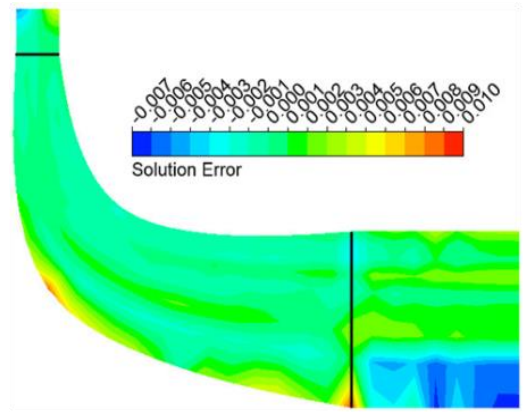

6)

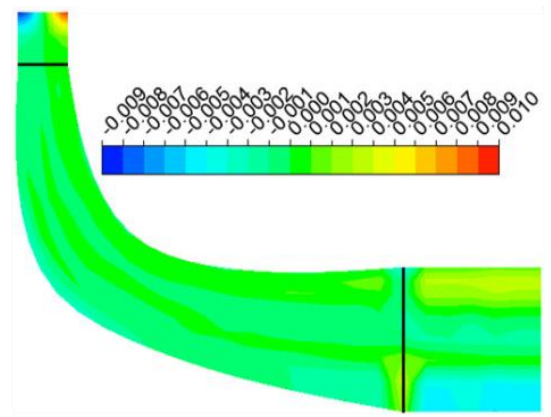

в)

Рисунок 7 - Контуры погрешности решения. Отношение выходного/входного радиусов ступицы газовой турбины: $а$ - 0.2; б - 0.3; в - 0.4. Значения коэффициента погрешности выражаются в процентах. 


\begin{tabular}{|c|c|c|c|c|c|c|}
\hline Impact Factor: & $\begin{array}{l}\text { ISRA (India) } \\
\text { ISI (Dubai, UAF } \\
\text { GIF (Australia) } \\
\text { JIF }\end{array}$ & $\begin{array}{l}=1.344 \\
=0.829 \\
=0.564 \\
=1.500\end{array}$ & $\begin{array}{l}\text { SIS (USA) } \\
\text { PИНЦ (Russia) } \\
\text { ESJI (KZ) } \\
\text { SJIF (Morocco) }\end{array}$ & $\begin{array}{l}=0.912 \\
=0.179 \\
=1.042 \\
=2.031\end{array}$ & $\begin{array}{l}\text { ICV (Poland) } \\
\text { PIF (India) }\end{array}$ & $\begin{array}{l}=6.630 \\
=1.940\end{array}$ \\
\hline
\end{tabular}

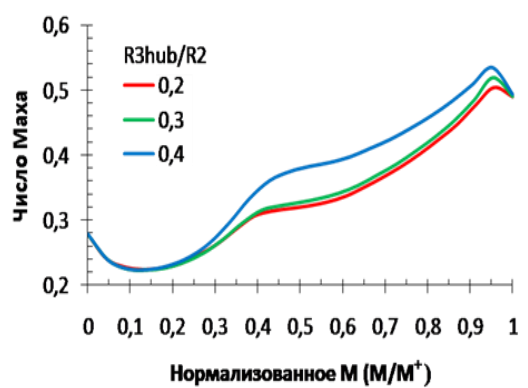

a)

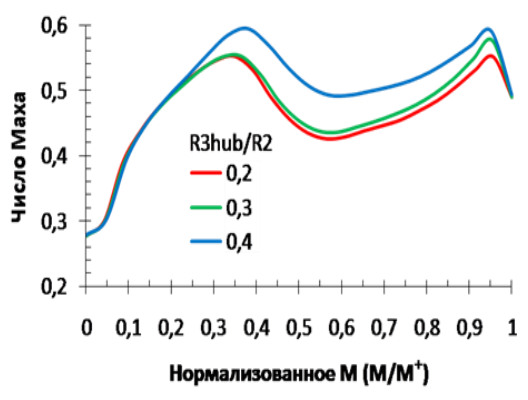

6)

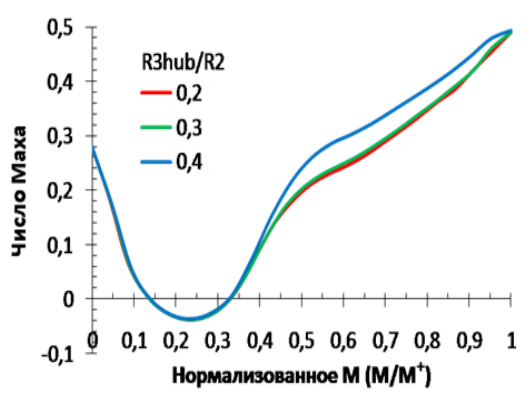

B)

Рисунок 8 - Величина числа Маха на линии воздушного потока (концевая кромка) от нормализованного М: а - средняя линия; б - сторона всасывания; в - сторона нагнетания.

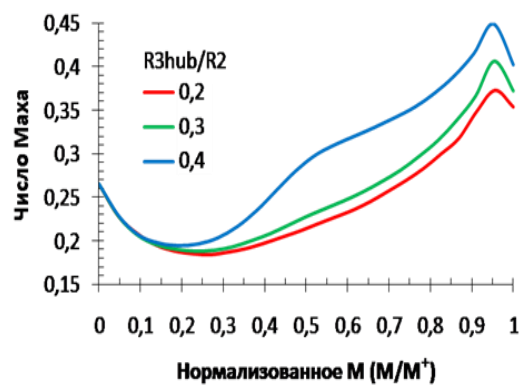

a)

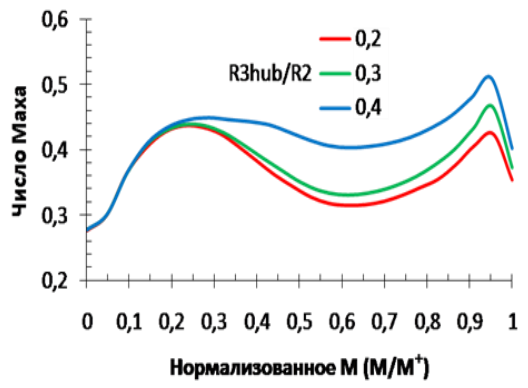

6)

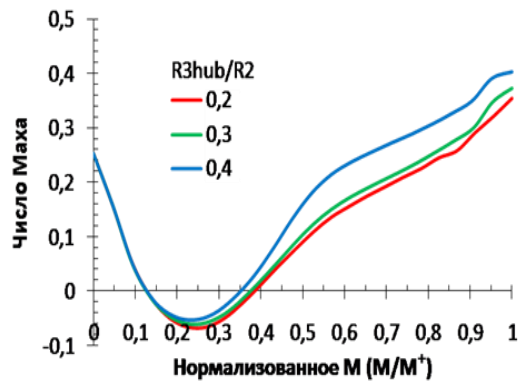

в)

Рисунок 9 - Величина числа Маха на линии воздушного потока (средняя часть) от нормализованного М: a - средняя линия; б - сторона всасывания; в - сторона нагнетания.

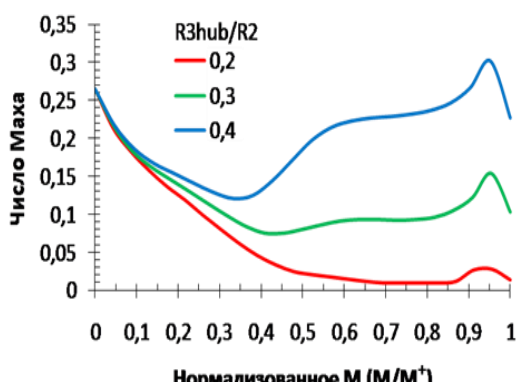

a)

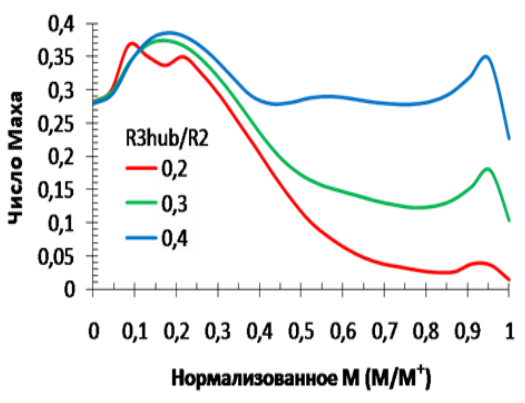

б)

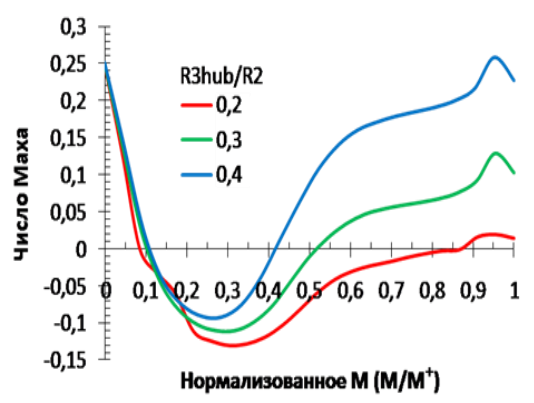

B)

Рисунок 10 - Величина числа Маха на линии воздушного потока (ступица) от нормализованного М: а - средняя линия; б - сторона всасывания; в - сторона нагнетания.

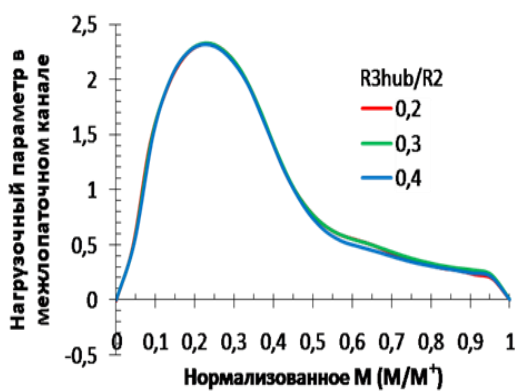

a)

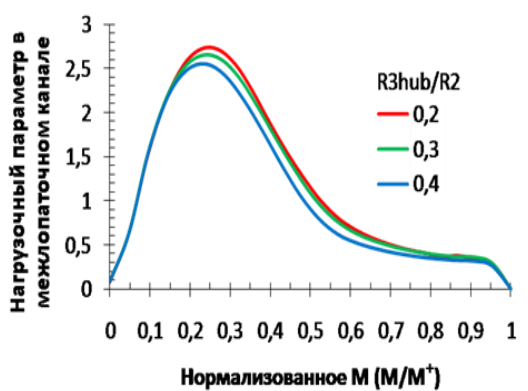

6)

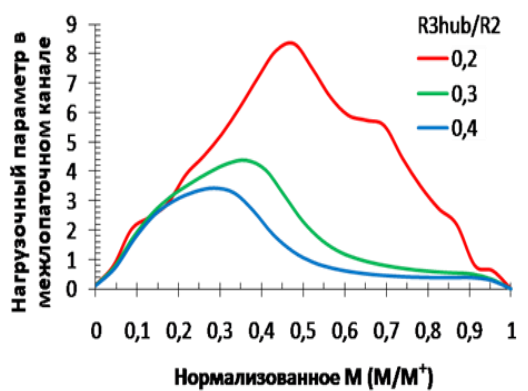

в)

Рисунок 11 - Величина нагрузочного параметра в межлопаточном канале (меридиональное сечение) от нормализованного М: а - концевая кромка; б - средняя часть; в - у ступицы. 


\begin{tabular}{l|lrl|l|ll} 
& ISRA (India) & $=\mathbf{1 . 3 4 4}$ & SIS (USA) & $=\mathbf{0 . 9 1 2}$ & ICV (Poland) & $=\mathbf{6 . 6 3 0}$ \\
Impact Factor: & ISI (Dubai, UAE) $=\mathbf{0 . 8 2 9}$ & PUHU (Russia) $=\mathbf{0 . 1 7 9}$ & PIF (India) & $=\mathbf{1 . 9 4 0}$ \\
& GIF (Australia) & $\mathbf{0 . 5 6 4}$ & ESJI (KZ) & $=\mathbf{1 . 0 4 2}$ & & \\
& JIF & $=\mathbf{1 . 5 0 0}$ & SJIF (Morocco) $=\mathbf{2 . 0 3 1}$ & &
\end{tabular}

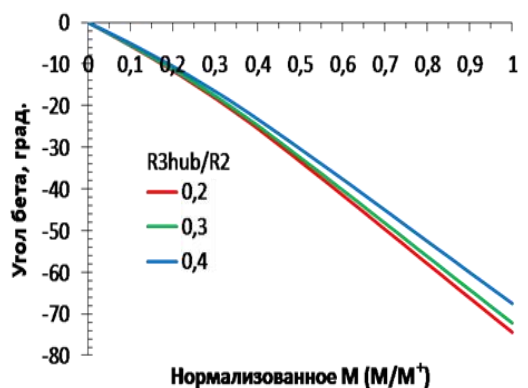

a)

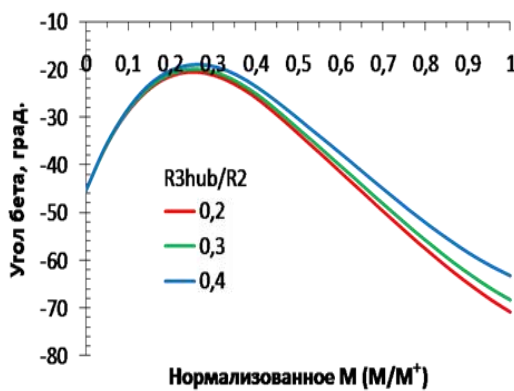

r)

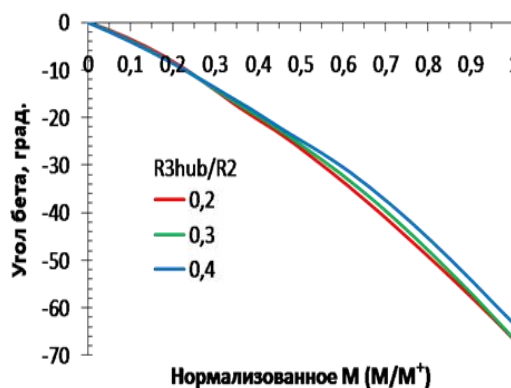

6)

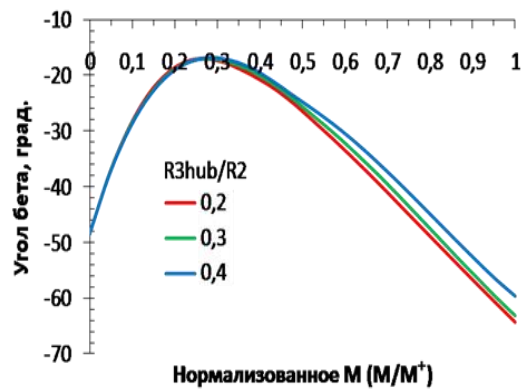

д)

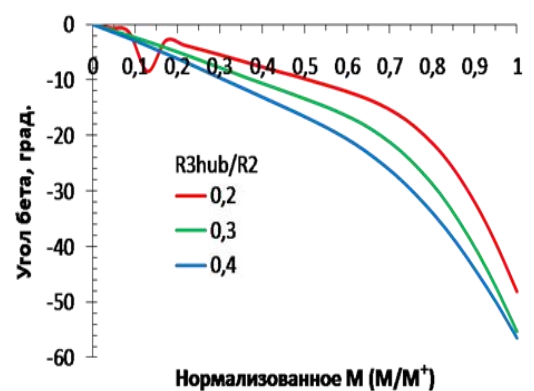

в)

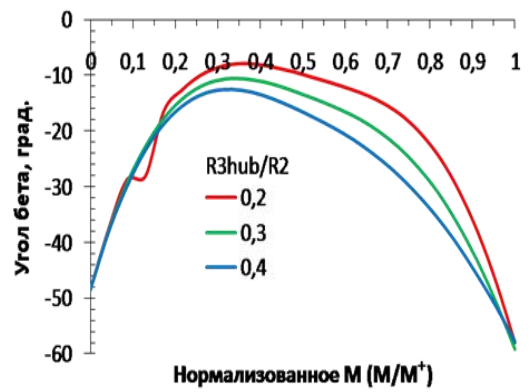

e)

Рисунок 12 - Величина углов бета лопасти газовой турбины и отклонения потока газа от нормализованного М: а - концевая кромка лопасти; б - средняя часть лопасти; в - лопасть у ступицы; г - угол потока на концевой кромке лопасти; д - угол потока в средней части лопасти; е угол потока у ступицы.

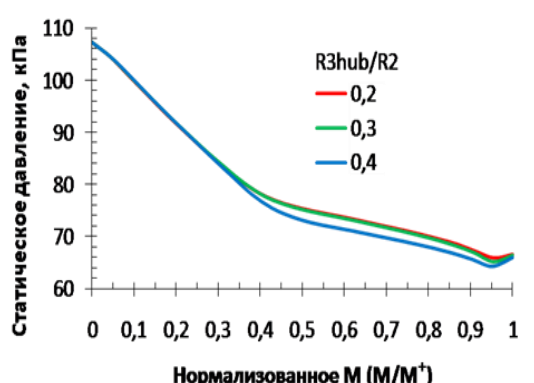

a)

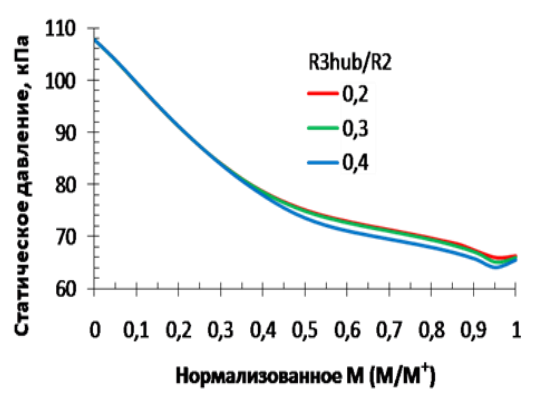

6)

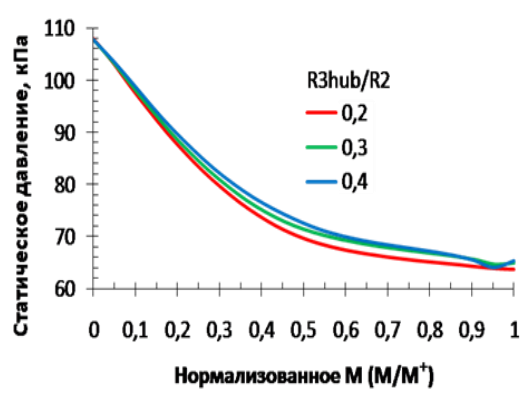

B)

Рисунок 13 - Величина статического давления в меридиональном сечении от нормализованного М: а - концевая кромка лопасти; б - средняя часть лопасти; в - лопасть у ступицы. 


\begin{tabular}{l|lrl|l|ll} 
& ISRA (India) & $=\mathbf{1 . 3 4 4}$ & SIS (USA) & $=\mathbf{0 . 9 1 2}$ & ICV (Poland) & $=\mathbf{6 . 6 3 0}$ \\
Impact Factor: & ISI (Dubai, UAE) $=\mathbf{0 . 8 2 9}$ & PUHU (Russia) $=\mathbf{0 . 1 7 9}$ & PIF (India) & $=\mathbf{1 . 9 4 0}$ \\
& GIF (Australia) & $\mathbf{0 . 5 6 4}$ & ESJI (KZ) & $=\mathbf{1 . 0 4 2}$ & & \\
& JIF & $=\mathbf{1 . 5 0 0}$ & SJIF (Morocco) $=\mathbf{2 . 0 3 1}$ & &
\end{tabular}

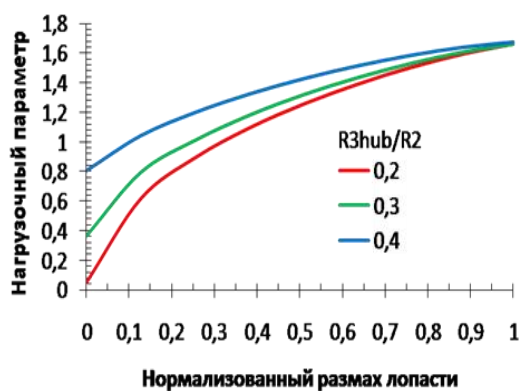

a)

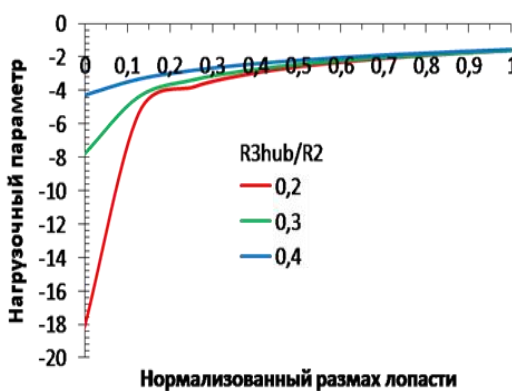

6)

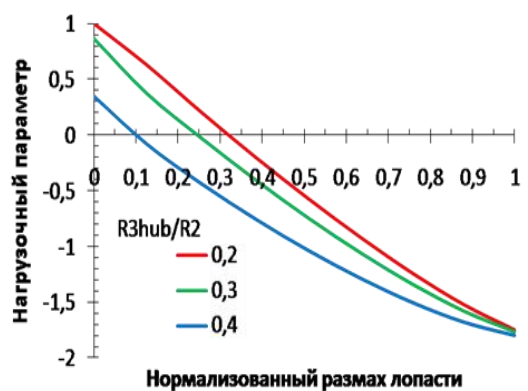

в)

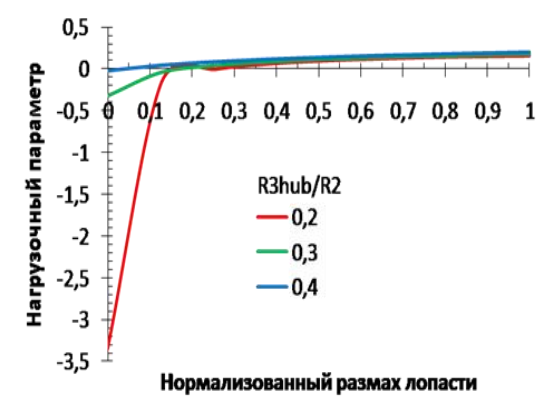

г)

Рисунок 14 - Величина нагрузочных параметров от нормализованного размаха лопасти газовой турбины: а - число де Халлера; б - лямбда; в - коэффициент восстановления идеального статического давления; г - параметр Цвайфеля.

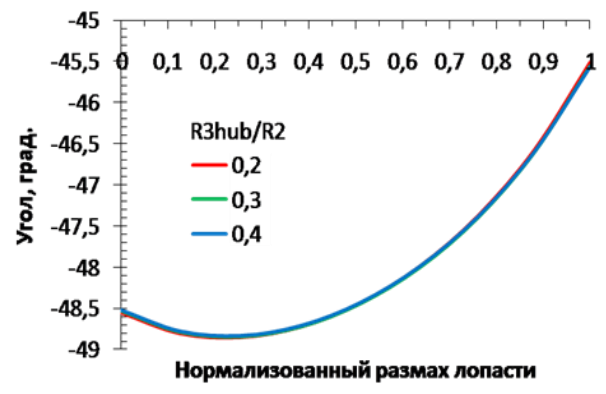

a)

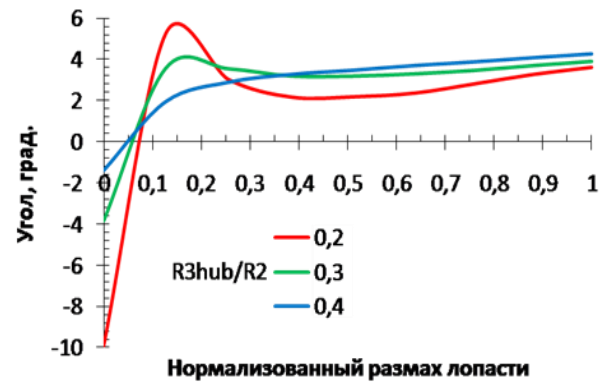

б)

Рисунок 15 - Величина углов падения (а) и отклонения (б) в меридиональном сечении от нормализованного размаха лопасти газовой турбины.

На основании проведенного анализа результатов компьютерного расчета параметров лопасти можно сделать следующие выводы о возможной эксплуатации радиальной газовой турбины при различных отношениях выходного/входного радиусов ступицы:

1. Для получения высокого КПД газовой турбины рекомендуется применять лопасти с параметрами, рассчитанными для R3hub/R2 = 0.2. Уменьшение температуры и давления газа при
R3hub/R2 = 0.4 приводит к снижению КПД газовой турбины.

2. С учетом высоких скоростей течения газа, действующих на рабочие лопасти газовой турбины при R3hub/R2 = 0.4, потери напора более значительны, чем у лопастей с другими аэродинамическими характеристиками.

3. Возможно уменьшение заданного количества лопастей газовой турбины при $\mathrm{R} 3 \mathrm{hub} / \mathrm{R} 2=0.2$, т. к. величина расчетного числа Цвайфеля в данном случае больше оптимального. 


\begin{tabular}{l|lrl|l|ll} 
& ISRA (India) & $=\mathbf{1 . 3 4 4}$ & SIS (USA) & $=\mathbf{0 . 9 1 2}$ & ICV (Poland) & $=\mathbf{6 . 6 3 0}$ \\
Impact Factor: & ISI (Dubai, UAE) $=\mathbf{0 . 8 2 9}$ & PUHIL (Russia) $=\mathbf{0 . 1 7 9}$ & PIF (India) & $=\mathbf{1 . 9 4 0}$ \\
& GIF (Australia) & $\mathbf{0 . 5 6 4}$ & ESJI (KZ) & $=\mathbf{1 . 0 4 2}$ & & \\
\hline JIF & $=\mathbf{1 . 5 0 0}$ & SJIF (Morocco) $=\mathbf{2 . 0 3 1}$ & & \\
\hline
\end{tabular}

\section{References:}

1. (2016) Gazovaya turbina. Available: http://enciklopediya-

tehniki.ru/promyshlennost-na-g/gazovayaturbina.html (Accessed: 23.02.2016).

2. (2016) Rabochie i napravlyayushchie lopatki turbiny. Available:

http://vdvizhke.ru/sudovye-parovyeturbiny/detali-sudovyh-parovyhturbin/rabochie-i-napravljajuwie-lopatkiturbiny.html (Accessed: 23.02.2016).

3. (2016) Konstruktsii lopatok turbin, tehnicheskie trebovaniya $\mathrm{k}$ konstruktsiyam, ih klassiphikatsiya. Available:

http://chiefengineer.ru/tehnologiimetaloobrabotki/tehnologii-obrabotkimetalla/konstrukcii-lopatok-turbin/ (Accessed: 23.02.2016).

4. (2016) Temperatura tormozheniya. Available: http://www.heuristic.su/effects/catalog/est/byI d/description/701/index.html (Accessed: 23.02.2016).

5. (2016) Chislo Maha. Available: http://nature.web.ru/db/msg.html?mid=11736 09 (Accessed: 23.02.2016).

6. (2016) Zakrutka potoka. Available: http://neftandgaz.ru/?p=4149 (Accessed: 23.02.2016).
7. (2016) Vybor razmera turbiny i chto takoe
$\mathrm{A} / \mathrm{R}$
turbiny.
Available: https://www.drive2.ru/b/1448570/ (Accessed: 23.02.2016)

8. (2016) Treugolniki (plany) skorostey. Available: http://helgior.livejournal.com/24729.html (Accessed: 23.02.2016).

9. (2016) Drosselirovanie gazov, parov i zhidkostey. Available: http://ispu.ru/files/u2/book2/TD2_1906/5.html (Accessed: 23.02.2016)

10. Johnson KE (2004) Adaptive Torque Control of Variable Speed Wind Turbines. National Renewable Energy Laboratory, Colorado. $94 \mathrm{p}$.

11. (2016) Teoreticheskaya harakteristika rabochego kolesa i ego osnovnye parametry. Available: http://msd.com.ua/vakuumnyeporshnevye-nasosy/teoreticheskayaxarakteristika-rabochego-kolesa-i-eeosnovnye-parametry/ (Accessed: 23.02.2016).

12. TurboSystem. Release 12.0. 2009 SAS IP, Inc. $-152 \mathrm{p}$. 